\title{
Universally Composable Client-to-Client General Authenticated Key Exchange
}

\author{
Haruki Ota,$^{\dagger}$ Kazuki Yoneyama,$^{\dagger \dagger}$ Shinsaku Kiyomoto, ${ }^{\dagger}$ \\ TOSHIAKI TANAKA ${ }^{\dagger}$ and KAZUO OHTA ${ }^{\dagger \dagger}$
}

\begin{abstract}
In large-scale networks, users want to be able to communicate securely with each other over a channel that is unreliable. When the existing 2- and 3-party protocols are realized in this situation, there are several problems: a client must hold many passwords and the load on the server concerning password management is heavy. In this paper, we define a new ideal client-to-client general authenticated key exchange functionality, where arbitrary 2-party key exchange protocols are applicable to protocols between the client and server and between servers. We also propose a client-to-client general authenticated key exchange protocol C2C-GAKE as a general form of the client-to-client model, and a client-to-client hybrid authenticated key exchange protocol C2C-HAKE as an example protocol of C2C-GAKE to solve the above problems. In C2C-HAKE, a server shares passwords only with clients in the same realm respectively, public/private keys are used between respective servers, and two clients between different realms share a final session key via the respective servers. Thus, with regard to password management in C2C-HAKE, the load on the server can be distributed to several servers. In addition, we prove that C2C-HAKE securely realizes the above functionality. C2CHAKE is the first client-to-client hybrid authenticated key exchange protocol that is secure in a universally composable framework with a security-preserving composition property.
\end{abstract}

\section{Introduction}

\subsection{Motivation}

In large-scale networks, users want to be able to communicate securely with each other over a channel that is unreliable, that is, they want to share a secure session key using a certain protocol. The simplest protocol is a 2party protocol, which is roughly classified into public key-based and secret key-based protocols. The users want to hold not public/private keys but secret keys, in particular, humanmemorable passwords. This is because passwords are used most widely as a personal authentication method at present, and it is easy for the users to use passwords. In the 2-party protocol, all users need to share respective passwords with each other, and the user must hold many passwords. A protocol that solves this problem is a 3-party protocol, in which the user (client) shares the password with a trusted server, and not with the other users (clients). However, there is also another problem on the server side in the 3-party protocol. That is, the server must manage all the passwords of clients such as registration, revocation and change. As the network scale becomes larger, the load on

$\dagger$ KDDI R\&D Laboratories, Inc.

$\dagger \dagger$ The University of Electro-Communications the server also becomes heavier. In order to solve this problem, several servers are used instead of one server, and are distributed to manage the clients. The load on one server can be distributed to several servers with regard to password management, since only the parent server of the clients manages their passwords. In such a protocol, a public/private key is used between the servers, while a password is used between the client and server. This is because it is more efficient in terms of scalability that the server holds a public/private key than that it shares respective passwords with all the other servers.

In order to satisfy practicality, client usability and server scalability requirements, Byun, Jeong, Lee and Park proposed a client-to-client password-authenticated key exchange proto-

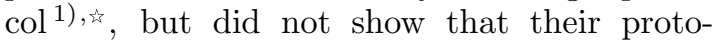
col is provably secure. In fact, it was shown that this protocol is not secure against various attacks ${ }^{2) ~ 4)}$. J. Kim, S. Kim, Kwak and Won improved the protocol of Byun, et al. ${ }^{4}$,

\footnotetext{
Strictly speaking, the client-to-client protocol may have a single-server setting or a cross-realm setting. The former means a 3-party protocol as described in this paper, and the latter means a 4-party protocol as defined later. In this paper, we refer only to the client-to-client protocol in the cross-realm setting as "client-to-client protocol".
} 
but attacks against this improved protocol were also presented ${ }^{5)}$. Then, Byun, Lee and $\operatorname{Lim}^{6)}$, and Yin and $\mathrm{Bao}^{7)}$, proposed provably secure client-to-client password-authenticated key exchange protocols, respectively. However, there is no client-to-client protocol whose security has been proven in a universally composable (UC) framework, as described below.

\subsection{Universal Composability}

Providing meaningful security guarantees under a composition with arbitrary protocols requires using an appropriate framework for representing and arguing such protocols. Canetti presented a general framework for representing cryptographic protocols such as key exchange protocols and analyzing their security ${ }^{8)}$. This framework allows the definition of the security properties of practically any cryptographic task in a unified and systematic way. In this framework, the security of protocols is maintained under a general protocol composition operation, which is called a universal composition, with an unbounded number of arbitrary protocol instances running concurrently in an adversarially controlled manner. Definitions of security in this framework are called universally composable. Canetti also proved the UC composition theorem, which states that the definition of UC-security achieves the goal of concurrent general composition.

Canetti and Krawczyk defined the security of a general key exchange protocol in the UC framework, and proved that an authenticated Diffie-Hellman (DH) key exchange protocol via digital signatures (SIGDH) is UC-secure ${ }^{9)}$. Canetti defined the security of a message authentication that realizes the secure MAC algorithm (MAC) in the UC framework ${ }^{10)}$. Canetti, Halevi, Katz, Lindell and MacKenzie defined the security of a password-based key exchange protocol, in particular security against off-line dictionary attack, in the UC framework, and proposed a UC-secure 2-party password-based key exchange protocol (UCPAKE) ${ }^{11)}$.

\subsection{Related Works}

Key exchange protocols are designed to provide a pair of users communicating over an unreliable channel with a secure session key. Bellare and Rogaway proposed indistinguishability-based formal models of security for a 2-party authenticated key exchange protocol $^{12)}$ and for a 3-party key distribution protocol ${ }^{13)}$, since such protocols were designed by trial and error. On the other hand, Bellare,
Canetti, and Krawczyk proposed a different approach to formal security models for a 2-party key exchange protocol, based on the multiparty simulatability paradigm ${ }^{14}$ ).

Among key exchange protocols, passwordbased authenticated key exchange protocols are more convenient and practical, since the users employ human-memorable passwords that are simpler to remember than cryptographic secret keys or public/private keys. However, such protocols are significantly subject to dictionary attacks due to the low entropy of passwords. Dictionary attacks are attacks in which an adversary tries a number of likely character strings (such as words, numbers and symbols in dictionaries) and all possible combinations of them in order to discover the passwords. A set of such character strings and their combinations is a so-called dictionary. Dictionary attacks are classified into on-line and off-line dictionary attacks. An on-line dictionary attack is an attack in which the adversary tries one password candidate from the dictionary at a time on-line to impersonate a client. An off-line dictionary attack is an attack in which the adversary records past communications, and searches for the password of a client that corresponds with the recorded communication off-line from the dictionary. The off-line dictionary attack is stronger than the on-line dictionary attack, which is unavoidable for protocols using passwords. The goal of password-based authenticated key exchange protocols is to eliminate only one password candidate from the dictionary at each trial in an on-line dictionary attack, since this attack can be prevented by limiting the number of passwords that are inputted incorrectly, for example.

The problem of the off-line dictionary attack for password-based authenticated key exchange protocols was mainly suggested by Bellovin and Merritt ${ }^{15)}$, which became the basis of considerable subsequent works in this area, but they provided only an informal security analysis and did not show that their protocol is provably secure. Halevi and Krawczyk proposed the first provably-secure password-based authentication protocol where a client authenticates using a password and a server using a public key ${ }^{16), 17)}$. Bellare, Pointcheval and Rogaway ${ }^{18)}$ then proposed a passwordbased authenticated key exchange protocol that is provably secure, based on definitions and proofs of Bellare and Rogaway ${ }^{12), 13)}$. Boyko, 
MacKenzie and Patel ${ }^{19)}$ also provided different proofs from those of Bellare, et al. ${ }^{18)}$ for a password-authenticated key exchange, based on definitions and proofs of Bellare, Canetti and Krawczyk $^{14)}$. Many password-based protocols were proposed in the 2-party case ${ }^{20) ~ 28)}$.

Abdalla, Fouque and Pointcheval proposed a password-based authenticated key exchange protocol in a 3 -party model ${ }^{29)}$ in which clients trying to establish a secret do not share a password between themselves but only with a trusted server. In this protocol, the two clients share the secret via the trusted server to exchange a secure session key. Also, the server is assumed to be honest but curious, in short, semi-honest. In other words, it honestly executes the protocol but wishes to know a final session key exchanged between clients in this protocol. Several password-based protocols have been proposed in the 3 -party case ${ }^{30), 31 \text { ). }}$.

\subsection{Contributions}

In this paper, we mainly provide the following three contributions:

Functionality. We define a new ideal clientto-client general authenticated key exchange functionality, where general means that arbitrary 2-party key exchange protocols are applicable to protocols between the client and server and between servers in the client-to-client model. Password-based key exchange functionality ${ }^{11)}$, authenticated key exchange functionality $^{9)}$ and message authentication functionality ${ }^{10)}$ were proposed as existing functionalities. However, these functionalities are realized in the 2-party model, and are not simply applicable to the client-to-client general authenticated key exchange functionality. This is because consistency between respective functionalities is required. Therefore, the proposed functionality is composed of a Setup phase and a MAC phase. The former is a phase for sharing MAC keys between the client and server and between servers, such as the password-based key exchange functionality and authenticated key exchange functionality. The latter is a phase for sending messages using the MAC key shared in the former phase, such as the message authentication functionality.

Protocol. Firstly, we propose a client-toclient general authenticated key exchange protocol C2C-GAKE as a general form of the clientto-client model. In C2C-GAKE, MAC keys are shared between each client and server and between servers using Setup protocols respec- tively, and then two clients exchange messages concerning a final session key using shared MAC keys. Secondly, we propose a client-toclient hybrid authenticated key exchange protocol C2C-HAKE as an example protocol of C2CGAKE, hybrid means that the password is used between the client and server, and the public/private key is used between servers, as described in Section 1.1. In C2C-HAKE, the UCsecure 2-party password-based key exchange protocol UCPAKE ${ }^{11)}$ and the UC-secure authenticated DH key exchange protocol via digital signatures SIGDH ${ }^{9)}$ are used between the client and server and between servers as the Setup protocols, respectively. Then, in C2CHAKE, two clients exchange DH-based public values as the messages concerning a final session key using the message authentication that realizes a secure MAC algorithm in the UC framework ${ }^{10)}$.

Security. We prove that C2C-HAKE securely realizes the above functionality using the security of the existing protocols and the UC composition theorem proven by Canetti ${ }^{8)}$, where the servers are assumed to be semi-honest, as well as the assumption of Abdalla, et al. ${ }^{29)}$. C2C-HAKE is the first client-to-client hybrid authenticated key exchange protocol that is secure in a UC framework with a security-preserving composition property.

\subsection{Organization}

The rest of this paper is organized as follows. We introduce some definitions used in this paper in Section 2. We define client-to-client general authenticated key exchange functionality in Section 3, and propose the client-to-client general authenticated key exchange protocol C2CGAKE and the client-to-client hybrid authenticated key exchange protocol C2C-HAKE respectively, in Section 4. We describe the UC composition theorem proven by Canetti, and prove the security of C2C-HAKE in the UC framework in Section 5. Finally, we present our conclusions in Section 6.

\section{Preliminaries}

\subsection{Communication Model}

This subsection introduces definitions used in this paper.

Protocol Participants. Each participant $U \in \mathbb{U}$ in this paper is either a client $C \in \mathbb{C}$ or a server $S \in \mathbb{S}$, while the set of participants $\mathbb{U}$ is a union $\mathbb{C} \cup \mathbb{S}$. We assume that the servers are semi-honest, in other words, they honestly 
execute the protocol but wish to know the final session key shared between clients, as well as the assumption of Abdalla, et al. ${ }^{29)}$.

Passwords. Each participant $U \in \mathbb{U}$ holds passwords $p w_{U}$ in the password-based protocol. A partner participant $P U \in \mathbb{U}$ of this participant $U$ holds vectors $p w_{P U}=\left\langle p w_{P U}[U]\right\rangle_{U \in \mathbb{U}}$ with an entry for each participant. $p w_{P U}[U]$ denotes the transformed-password of $U$ by $P U$, as defined in Ref. 29). In a symmetric model, $p w_{P U}[U]=p w_{U}$, and we assume the symmetric model in this paper.

Remark 1: We assume that servers are semihonest, as described above. The reason for this is as follows: The server forwards messages sent by clients without altering, delaying, or intercepting them, as described later. If the server is assumed to be malicious, attacks by this malicious server cannot be prevented. Hence, the server must honestly execute the protocol, and we must assume that the servers are semihonest.

Notations. Let $p$ be a prime and let $g$ be a generator of a large subgroup of the prime order $q$ in $\mathbb{Z}_{p}^{*}$, where $q \mid p-1$. For simplicity, we omit " $(\bmod p) "$ in this paper when computing the modular exponentiation.

\subsection{UC Framework}

This subsection briefly describes the UC framework proposed by Canetti ${ }^{8)}$.

The security requirements of a given task are captured via a set of instructions for a "trusted party" that obtains the inputs of the participants and provides them with the desired outputs. Here, we consider an ideal process by which the participants hand their inputs to the trusted party and obtain their outputs from it, without any other interaction. We say that a protocol securely carries out the given task, if running the protocol amounts to "emulating" the ideal process. We call the algorithm run by the trusted party an (ideal) functionality.

The model of computation includes the participants running the protocol, an adversary $\mathcal{A}$ and an environment $\mathcal{Z}$ that is an additional adversarial entity. The environment $\mathcal{Z}$ generates the inputs to all participants, and reads all outputs. In addition, it interacts with the adversary in an arbitrary way throughout the computation. A protocol is said to securely realize a given functionality $\mathcal{F}$ if for any adversary $\mathcal{A}$ there exists a "simulator" $\mathcal{S}$, such that no environment $\mathcal{Z}$ can distinguish whether it is interacting with $\mathcal{A}$ and participants running the protocol, or with $\mathcal{S}$ and participants that interact with $\mathcal{F}$ in the ideal process.

\section{Functionality}

This section defines a new ideal client-toclient general authenticated key exchange functionality, and explains notable points concerning this functionality.

We define the client-to-client general authenticated key exchange functionality $\mathcal{F}_{\mathrm{C} 2 \mathrm{C}-\mathrm{GAKE}}$ for the client-to-client general authenticated key exchange protocol where arbitrary 2-party key exchange protocols are applicable to protocols between the client and server and between servers. Figures 1 and $\mathbf{2}$ show the functionality $\mathcal{F}_{\mathrm{C} 2 \mathrm{C}-\mathrm{GAKE}}$.

Notable Points. We explain notable points concerning the functionality $\mathcal{F}_{\mathrm{C} 2 \mathrm{C}-\mathrm{GAKE}}$. This functionality needs to be defined such that a final session key shared between two clients is not known to all servers, and the MAC is adequate in order to prevent messages sent between participants from being altered, when the final session key is shared. The MAC key also requires to be shared between the participants in order to use the MAC. Thus, the functionality is classified into two phases: Setup phase and MAC phase. The former is a phase for sharing the MAC key between participants, and the latter is a phase for sending messages using the MAC key shared in the former phase. The Setup phase is called several times in this functionality, according to the protocol specification. Information obtained in the Setup phase is transmitted to the MAC phase. The MAC phase is also called several times in this functionality, according to the protocol specification. Information obtained in the MAC phase is transmitted to another MAC phase. In the Setup phase, this functionality is defined such as password-based key exchange functionality $\mathcal{F}_{\text {pwKE }}{ }^{11)}$ when a value NewSession has a password as the argument. On the other hand, in this phase, this functionality is defined such as authenticated key exchange functionality $\mathcal{F}_{\mathrm{KE}}{ }^{9)}$ when a value NewSession does not include a password in the argument. The functionality $\mathcal{F}_{\mathrm{C} 2 \mathrm{C}-\mathrm{GAKE}}$ in both cases plays essentially the same role, since this functionality receives NewSession from the participant, obtains the session key, and sends it to the participant. However, there are the following two differences between the two cases:

- One of the uncorrupted participants may 


\section{Functionality $\mathcal{F}_{\mathrm{C} 2 \mathrm{C}-\mathrm{GAKE}}$}

The functionality $\mathcal{F}_{\mathrm{C} 2 \mathrm{C}-\mathrm{GAKE}}$ proceeds as follows, running on security parameter $k$, with participants $U_{1}, \ldots, U_{n}(n \in \mathbb{N})$ and an adversary.

- Setup phase:

(1) Upon receiving a value (NewSession, sid, $U_{i}, U_{j},(p w$,$) role) from participant U_{i}$ :

Record $\left(U_{i}, U_{j}(, p w)\right)$, mark this record fresh, and send (NewSession, sid, $U_{i}, U_{j}$, role) to the adversary (usual execution).

(2) Upon receiving a value (TestPwd, sid, $U_{i}, p w^{\prime \prime}$ ) from the adversary:

If there is a record of the form $\left(U_{i}, U_{j}, p w\right)$ which is fresh, then do: If $p w=p w^{\prime \prime}$, mark the record compromised and reply to the adversary with "correct guess". If $p w \neq p w^{\prime \prime}$, mark the record interrupted and reply to the adversary with "wrong guess" (unusual execution, notable point (1)).

(3) Upon receiving a value (NewKey, sid, $U_{i}, s k$ ) from the adversary:

If there is a record of the form $\left(U_{i}, U_{j}(, p w)\right)$, then record $\left(U_{i}, U_{j},(p w) s k,\right)$. In addition, if there is already a recorded tuple $\left(U_{j}, U_{i},\left(p w^{\prime},\right) s k^{\prime}\right)$, then proceed as follows:

(a) If either $\left(U_{i}, U_{j}, p w\right)$ or $\left(U_{j}, U_{i}, p w^{\prime}\right)$ is compromised, or either $U_{i}$ or $U_{j}$ is corrupted, then send messages $(s i d, s k)$ to $U_{i}$ and $\left(s i d, s k^{\prime}\right)$ to $U_{j}$, respectively (unusual execution, notable point (2)).

(b) Otherwise, proceed as follows:

(i) If either $\left(U_{i}, U_{j}, p w\right)$ or $\left(U_{j}, U_{i}, p w^{\prime}\right)$ is interrupted, or $p w \neq p w^{\prime}$, then record $\left(U_{i}, U_{j}, \neq\right)$, choose random session keys $s k^{\prime \prime}$ and $s k^{\prime \prime \prime}$, and send $\left(s i d, s k^{\prime \prime}\right)$ to $U_{i}$ and $\left(s i d, s k^{\prime \prime \prime}\right)$ to $U_{j}$, respectively (unusual execution, notable point (3)).

(ii ) Otherwise, then choose a random session key $s k^{\prime \prime}$, and send ( $\left.s i d, s k^{\prime \prime}\right)$ to $U_{i}$ and $U_{j}$, respectively (usual execution).

Either way, mark the record $\left(U_{i}, U_{j}(, p w)\right)$ as completed.

Fig. 1 Setup phase of functionality $\mathcal{F}_{\mathrm{C} 2 \mathrm{C}-\mathrm{GAKE}}$.

input an incorrect password by mistake ${ }^{\text {is }}$.

- The adversary is given the ability to carry out the password guessing attack.

Then, we explain six notable points in situations of unusual execution and unusual halt with regard to the functionality $\mathcal{F}_{\mathrm{C} 2 \mathrm{C}-\mathrm{GAKE}}$.

(1) In the Setup phase, the password guessing attack by the adversary needs to be carried out when a value NewSession has a password as the argument. If the adversary is successful in guessing the password, the session is marked compromised. Otherwise, the session is marked interrupted.

(2) If the session is compromised, or either of the participants is corrupted in the Setup phase, both participants use a random key selected by the adversary such that the protocol continues correctly. In order to make the participants use the random key, the functionality sends the random key received from the adversary to these participants, respectively.

\footnotetext{
许 A participant frequently inputs an incorrect password by mistake, although he/she rarely uses an incorrect private key by mistake (due to a software bug for example). Thus, in this paper, we do not assume that the participant uses incorrect private keys by mistake.
}

(3) When session keys shared between both participants are different in the Setup phase due to the mistaken input of an incorrect password or a failed password guessing attack by the adversary, the protocol halts in the MAC phase, since later MAC verification ends in failure. In order to halt the protocol in the MAC phase, information $\left(U_{i}, U_{j}, \neq\right)$ that the two passwords are different or the session is interrupted is recorded in the Setup phase.

(4) When a session key shared in the Setup phase is different from a session key inputted from the same participant in the MAC phase $\left(s k \neq s k^{\prime}\right)$, the protocol halts, since later $\mathrm{MAC}$ verification ends in failure. In order to compare these two session keys, one session key is recorded in the Setup phase and the other session key is inputted from the same participant in the MAC phase.

(5) If either of the participants is corrupted in the MAC phase and the received participant is not a partner one, then the protocol halts, since the message the adversary has altered is sent to an unintended participant.

(6) If either of the participants is corrupted in the MAC phase and the received partici- 


\section{Functionality $\mathcal{F}_{\mathrm{C} 2 \mathrm{C}-\mathrm{GAKE}}$}

- MAC phase:

(1) Upon receiving (send, sid, $U_{j}, m, s k^{\prime}$ ) from participant $U_{i}$ :

Proceed as follows:

(a) If $\left(U_{i}, U_{j},(p w) s k,\right)$ is not recorded, $s k \neq s k^{\prime}$ or $\left(U_{i}, U_{j}, \neq\right)$ is recorded, then halt (unusual halt, notable points (3) and (4)).

(b) If $s k=s k^{\prime}$, then record $\left(s i d, U_{i}, U_{j}, m\right)$, and send (sent, sid, $\left.U_{i}, U_{j}, m\right)$ to the adversary (usual execution).

(2) Upon receiving (send, sid, $U_{j}^{\prime}, m^{\prime}$ ) from the adversary:

Proceed as follows:

(a) If $U_{i}$ is corrupted, then proceed as follows:

(i) If $U_{j}^{\prime} \neq U_{j}$, then output (sent, sid, $U_{i}, m^{\prime}$ ) to participant $U_{j}^{\prime}$, and halt (unusual halt, notable point (5)).

(ii) If $U_{j}^{\prime}=U_{j}$, then output (sent, sid, $U_{i}, m^{\prime}$ ) to participant $U_{j}$ (unusual execution, notable point (6)).

(b) If $U_{i}$ is uncorrupted, then proceed as follows:

(i) If there is already a recorded tuple (sent, sid, $U_{i}^{\prime \prime}, U_{j}^{\prime \prime}, m^{\prime \prime}$ ), and both $U_{j}$ and $U_{j}^{\prime \prime}$ are clients, then output (sent, sid, $U_{i}, m$ ) to participant $U_{j}$ and halt (usual halt).

(ii) Otherwise, then record (sent, sid, $U_{i}, U_{j}, m$ ) and output (sent, sid, $U_{i}, m$ ) to participant $U_{j}$ (usual execution).

Fig. 2 MAC phase of functionality $\mathcal{F}_{\mathrm{C} 2 \mathrm{C}-\mathrm{GAKE}}$.

pant is a partner one, then the messages the adversary has altered are sent to the partner participant such that the protocol continues correctly is.

\section{Proposed Protocol}

This section proposes a client-to-client general authenticated key exchange protocol C2CGAKE as a general form of the client-to-client model, and a client-to-client hybrid authenticated key exchange protocol C2C-HAKE as an example protocol of C2C-GAKE.

Firstly, we propose a client-to-client general authenticated key exchange protocol C2CGAKE. C2C-GAKE is composed of Setup protocols and MAC protocols, as shown in Fig. 3. Setup $(s k)$ denotes that a key $s k$ is shared between both participants using Setup protocol, and $\operatorname{MAC}(s k, m)$ denotes that a message $m$ and a MAC of $m$ using a key $s k$ are sent from one participant to another participant. The participant that receives the message $m$ and its MAC verifies them, and if this verification fails, the protocol halts.

\footnotetext{
The MAC phase of this functionality is defined such as message authentication functionality $\mathcal{F}_{\text {AUTH }} 10$ ) described later. In the functionality $\mathcal{F}_{\text {AUTH }}$, only the instance of notable point (5) is defined. However, in this paper, we also assume the instance of this notable point in order to take more possibilities into consideration, hereafter.
}

Secondly, we propose a client-to-client hybrid authenticated key exchange protocol C2CHAKE. C2C-HAKE is composed of the UCsecure 2-party password-based key exchange protocol UCPAKE ${ }^{11)}$, the UC-secure authenticated DH key exchange protocol via digital signatures SIGDH ${ }^{9}$, and the message authentication that realizes the secure MAC algorithm in the UC framework ${ }^{10)}$, as shown in Fig. 4. $\operatorname{UCPAKE}(s k)$ and $\operatorname{SIGDH}(s k)$ denote that a key $s k$ is shared between both participants using UCPAKE and SIGDH, respectively. $\operatorname{MAC}(s k, m)$ and its MAC verification are the same as those of C2C-GAKE.

We construct protocol C2C-HAKE, which securely realizes functionality $\mathcal{F}_{\mathrm{C} 2 \mathrm{C}-\mathrm{GAKE}}$ in the $\left(\mathcal{F}_{\text {pwKE }}, \mathcal{F}_{\mathrm{KE}}, \mathcal{F}_{\mathrm{AUTH}}\right)$-hybrid model 的的, where $\mathcal{F}_{\text {AUTH }}$ denotes message authentication functionality ${ }^{10)}$.

(1) A client $A$ and a server $S_{1}$ send (NewSession, sid, $A, S_{1}, p w_{A}$, initiator) and (NewSession, sid, $S_{1}, A, p w_{A}$, responder) to the functionality $\mathcal{F}_{\text {pwKE }}$ respectively, and then receive $\left(s i d, s k_{A}\right)$ from $\mathcal{F}_{\text {pwKE }}$ respectively.

\footnotetext{
败 From the first, $\mathcal{F}_{\mathrm{KE}}$ in Lemma 2 described later is defined in Ref. 9). On the other hand, the definition of $\mathcal{F}_{\mathrm{KE}}$ in Ref. 11) is somewhat different from the one in Ref. 9), although the differences are inconsequential. In this paper, we use the definition of $\mathcal{F}_{\mathrm{KE}}$ in Ref. 11) for consistency with $\mathcal{F}_{\text {pwKE }}$, hereafter.
} 


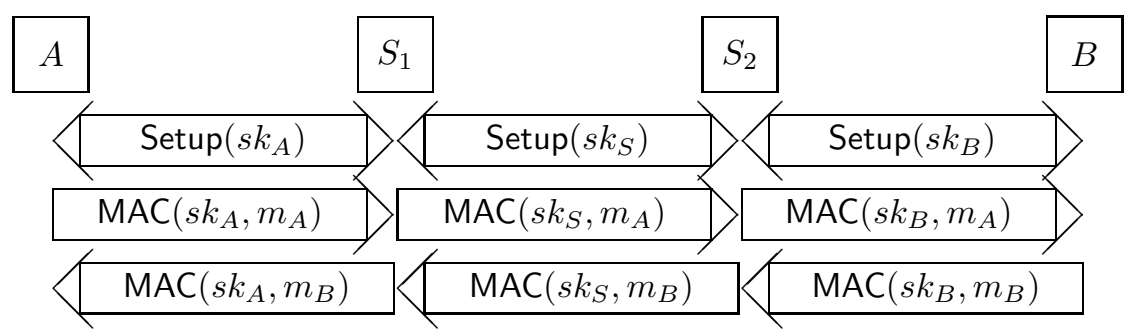

Fig. 3 Client-to-client general authenticated key exchange protocol C2C-GAKE.

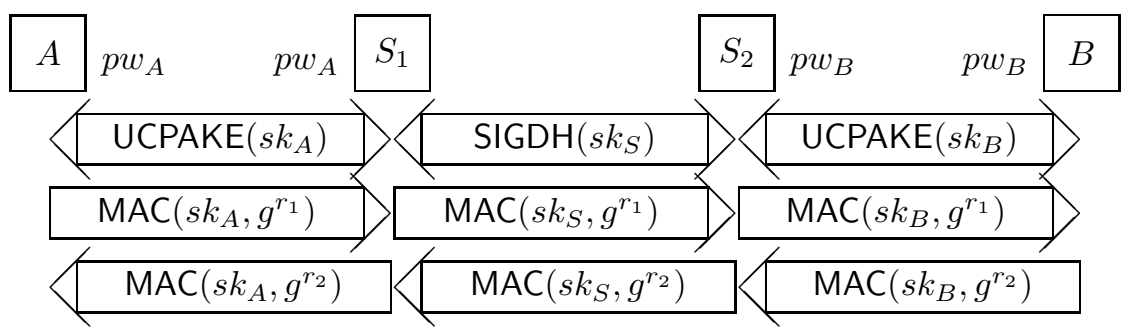

Fig. 4 An example C2C-HAKE of the proposed protocol C2C-GAKE.

(2) A client $B$ and a server $S_{2}$ send (NewSession, sid, $B, S_{2}, p w_{B}$, initiator) and (NewSession, sid, $S_{2}, B, p w_{B}$, responder) to $\mathcal{F}_{\text {pwKE }}$ respectively, and then receive (sid, $\left.s k_{B}\right)$ from $\mathcal{F}_{\text {pwKE }}$ respectively.

(3) $S_{1}$ and $S_{2}$ send (NewSession, sid, $S_{1}$, $S_{2}$, initiator) and (NewSession, sid, $S_{2}, S_{1}$, responder) to the functionality $\mathcal{F}_{\mathrm{KE}}$ respectively, and then receive $\left(s i d, s k_{S}\right)$ from $\mathcal{F}_{\mathrm{KE}}$ respectively.

(4) $A$ (resp. B) initially selects a random value $r_{1} \in \mathbb{Z}_{q}$ (resp. $r_{2} \in \mathbb{Z}_{q}$ ), sends (send, sid, $S_{1}, g^{r_{1}}$ ) (resp. (send, sid, $\left.S_{2}, g^{r_{2}}\right)$ ) to the functionality $\mathcal{F}_{\text {AUTH }}$, and then $S_{1}$ (resp. $S_{2}$ ) receives (sent, sid, $A, g^{r_{1}}$ ) (resp. (sent, sid, $\left.B, g^{r_{2}}\right)$ ) from $\mathcal{F}_{\text {AUTH }}$.

(5) $S_{1}$ (resp. $S_{2}$ ) sends (send, sid, $S_{2}, g^{r_{1}}$ ) (resp. (send, sid, $\left.S_{1}, g^{r_{2}}\right)$ ) to $\mathcal{F}_{\mathrm{AUTH}}$, and then $S_{2}$ (resp. $S_{1}$ ) receives (sent, sid, $S_{1}, g^{r_{1}}$ ) (resp. (sent, sid, $\left.S_{2}, g^{r_{2}}\right)$ ) from $\mathcal{F}_{\text {AUTH }}$.

(6) $S_{2}$ (resp. $S_{1}$ ) sends (send, sid, $B, g^{r_{1}}$ ) (resp. (send, sid, $\left.A, g^{r_{2}}\right)$ ) to $\mathcal{F}_{\mathrm{AUTH}}$, and then $B$ (resp. $A$ ) receives (sent, sid, $S_{2}, g^{r_{1}}$ ) (resp. (sent, sid, $\left.S_{1}, g^{r_{2}}\right)$ ) from $\mathcal{F}_{\text {AUTH }}$.

If the protocol has been run correctly, $A$ and $B$ share a final session key $g^{r_{1} r_{2}}$.

\section{Security}

\subsection{UC Composition Theorem}

This subsection introduces the security of the existing protocols and the UC composition theorem proven by Canetti ${ }^{8)}$.

The Lemmas 1 and 2 state that UCPAKE and SIGDH are secure in the UC framework, respectively.

Lemma 1 (Ref. 11) [Theorem 1]) Let $\mathcal{F}_{\text {crs }}$ be the ideal functionality that provides a common reference string to all participants. Assume that $E$ is a CCA-secure labeled encryption scheme, that $E^{\prime}$ is a CPA-secure encryption scheme, that $\mathcal{H}$ is a family of smooth projective hash functions with respect to $E$, that $\mathcal{H}^{\prime}$ is a family of smooth projective hash functions with respect to $E^{\prime}$, that the proof system is simulation-sound zero-knowledge, and that the one-time signature scheme is secure. Then the protocol UCPAKE securely realizes the functionality $\mathcal{F}_{\text {pwKE }}$ in the $\mathcal{F}_{\text {crs }}$-hybrid model, in the presence of static-corruption adversaries.

Lemma 2 (Ref. 9) [Theorem 9]) Let $\mathcal{F}_{\text {SIG }}$ be the ideal signature functionality. If the Decisional DH (DDH) assumption holds, then the protocol SIGDH securely realizes the functionality $\mathcal{F}_{\mathrm{KE}}$ in the $\mathcal{F}_{\mathrm{SIG}}$-hybrid model.

The following lemma denotes the UC composition theorem, which states that the definition

\footnotetext{
In Theorem 1 of Ref. 11), UCPAKE securely realizes not only $\mathcal{F}_{\text {pwKE }}$ but also its multi-session extension $\hat{\mathcal{F}}_{\text {pwKE }}$. However, we consider only $\mathcal{F}_{\text {pwKE }}$ for simplicity.
} 
of UC-security achieves the goal of concurrent general composition.

Lemma 3 (Refs. 8), 9)) Let $\mathcal{F}, \mathcal{G}$ be ideal functionalities. Let $\pi$ be an $n$-party protocol in the $\mathcal{F}$-hybrid model, and let $\rho$ be an $n$ party protocol that securely realizes $\mathcal{F}$ in the $\mathcal{G}$-hybrid model. Then the protocol $\pi^{\rho}$ in the $\mathcal{G}$-hybrid model emulates the protocol $\pi$ in the $\mathcal{F}$-hybrid model. In particular, if $\pi$ securely realizes some ideal functionality $\mathcal{I}$ in the $\mathcal{F}$ hybrid model, then $\pi^{\rho}$ securely realizes $\mathcal{I}$ in the $\mathcal{G}$-hybrid model.

The proofs of these lemmas are omitted due to lack of space. Refer to the corresponding literature for the details of the respective proofs.

From the UC composition theorem of Lemma 3, the proposed protocol C2C-HAKE emulates the protocols (UCPAKE, SIGDH, $\mathrm{MAC})$ in the $\left(\mathcal{F}_{\mathrm{pwKE}}, \mathcal{F}_{\mathrm{KE}}, \mathcal{F}_{\mathrm{AUTH}}\right)$-hybrid model. That is, C2C-HAKE satisfies the security required by $\left(\mathcal{F}_{\mathrm{pwKE}}, \mathcal{F}_{\mathrm{KE}}, \mathcal{F}_{\mathrm{AUTH}}\right)$. However, these functionalities do not satisfy all the security required for the client-to-client protocols, such as C2C-HAKE, since they are defined such that only the security for the 2-party protocols is satisfied. Generally, all the security for any protocols is not satisfied just because some functionality is securely realized in the UC framework. When some protocol securely realizes some functionality in the UC framework, this protocol satisfies only the security required by this functionality, but does not always satisfy the security not guaranteed by this functionality. Hence, the new functionality $\mathcal{F}_{\mathrm{C} 2 \mathrm{C}-\mathrm{GAKE}}$ that satisfies the security for the client-to-client protocols needs to be defined, and we prove that the proposed protocol C2C-HAKE securely realizes this functionality. Here, we show that the existing functionalities $\left(\mathcal{F}_{\text {pwKE }}, \mathcal{F}_{\text {KE }}, \mathcal{F}_{\text {AUTH }}\right)$ do not satisfy the security required for the specific attack on the client-to-client protocol.

As described in the notable points (3) and (4) of the functionality $\mathcal{F}_{\mathrm{C} 2 \mathrm{C}-\text { GAKE }}$, the protocol halts in the MAC phase, when session keys shared between both participants are different in the Setup phase due to the mistaken input of an incorrect password or a failed password guessing attack by the adversary, or when a session key shared in the Setup phase is different from a session key inputted by the same participant in the MAC phase $\left(s k \neq s k^{\prime}\right)$. Therefore, in the case of the proposed functionality $\mathcal{F}_{\mathrm{C} 2 \mathrm{C}-\mathrm{GAKE}}$, the environment $\mathcal{Z}$ can- not distinguish whether it is interacting with $\mathcal{A}$ and participants running the protocol in the $\left(\mathcal{F}_{\text {pwKE }}, \mathcal{F}_{\text {KE }}, \mathcal{F}_{\text {AUTH }}\right)$-hybrid model or with $\mathcal{S}$ and participants in the ideal process, as proven later. However, in the existing functionalities $\left(\mathcal{F}_{\text {pwKE }}, \mathcal{F}_{\mathrm{KE}}, \mathcal{F}_{\mathrm{AUTH}}\right)$, when session keys shared between both participants are different in $\mathcal{F}_{\text {pwKE }}$ due to the mistaken input of an incorrect password or a failed password guessing attack by the adversary, the information that two session keys are different cannot be reported in $\mathcal{F}_{\text {AUTH }}$. Also, when a session key shared in $\mathcal{F}_{\text {pwKE }}$ or $\mathcal{F}_{\mathrm{KE}}$ is different from a session key given to the same participant in $\mathcal{F}_{\text {AUTH }}$, both session keys cannot be compared in $\mathcal{F}_{\text {AUTH }}$. Therefore, in the case of the existing functionalities $\left(\mathcal{F}_{\text {pwKE }}, \mathcal{F}_{\mathrm{KE}}, \mathcal{F}_{\mathrm{AUTH}}\right)$, the environment $\mathcal{Z}$ can distinguish whether it is interacting with $\mathcal{A}$ and participants running the real protocol or with $\mathcal{S}$ and participants in the ideal process. Consequently, the existing functionalities do not satisfy the security required for the specific attack on the client-to-client protocol.

In the next subsection, we prove the security of C2C-HAKE.

\subsection{Main Theorem}

This subsection proves the security of C2CHAKE using the functionality $\mathcal{F}_{\mathrm{C} 2 \mathrm{C}-\mathrm{GAKE}}$ and Lemmas $1-3$.

The following theorem states that C2C-HAKE is a UC-secure client-to-client hybrid authenticated key exchange protocol as long as the DDH assumption holds and the underlying protocols it uses are UC-secure.

Theorem 1 If the DDH assumption holds and the servers are semi-honest, then the protocol C2C-HAKE securely realizes the functionality $\mathcal{F}_{\mathrm{C} 2 \mathrm{C}-\text { GAKE }}$ in the $\left(\mathcal{F}_{\mathrm{pwKE}}, \mathcal{F}_{\mathrm{KE}}, \mathcal{F}_{\mathrm{AUTH}}\right)$ hybrid model.

Notable Points. We explain some notable points concerning the validity of the simulation in the proof of Theorem 1. The clients can be actively corrupted by an adversary, while the semi-honest servers are only passively corrupted. Hereafter, we refer to the protocol in the $\left(\mathcal{F}_{\text {pwKE }}, \mathcal{F}_{\text {KE }}, \mathcal{F}_{\text {AUTH }}\right)$-hybrid model as $\mathcal{F}$ hybrid real protocol. Also, henceforth, $C$ denotes $A$ or $B, S$ denotes $S_{1}$ or $S_{2}$, and $r$ denotes $r_{1}$ chosen by $A$ or $r_{2}$ chosen by $B$, respectively.

- If both clients are uncorrupted, a perfect simulation can be executed in a natural way, but there is one notable point. Participants may share different session keys by the definition of $\mathcal{F}_{\text {pwKE }}$ in the UCPAKE phase, since 
one of them has inputted the incorrect password by mistake or the password guessing attack by the adversary has failed. In this case, the session key of each participant shared in the $\mathcal{F}$-hybrid real protocol must be the same as that in the ideal process.

- If the password guessing attack by the adversary has been successful or either of the clients is corrupted, there are the following two notable points according to the phases in which the corruption occurs or the successful password guessing attack by the adversary is carried out:

- UCPAKE phase between $C$ and $S$ :

By the definition of $\mathcal{F}_{\mathrm{C} 2 \mathrm{C}-\mathrm{GAKE}}, C$ and $S$ use the same session key generated by an adversary both in the $\mathcal{F}$-hybrid real protocol and in the ideal process, even if the password shared by $C$ and $S$ is successfully guessed or $C$ is corrupted. Hence, whether the MAC verification is successful in the phase in which this session key is used, is the same both in the $\mathcal{F}$-hybrid real protocol and in the ideal process.

- MAC phase from $C$ to $S$ :

By the definition of $\mathcal{F}_{\mathrm{C} 2 \mathrm{C}-\mathrm{GAKE}}$, if $C$ is corrupted and the received participant is not $S, S$ cannot receive the message related to the final session key, both in the $\mathcal{F}$-hybrid real protocol and in the ideal process. Both the $\mathcal{F}$-hybrid real protocol and the ideal process halt in this MAC phase, since the message the adversary has altered is sent to an unintended participant. On the other hand, if $C$ is corrupted and the received participant is $S, S$ receives the message the adversary has altered, both in the $\mathcal{F}$ hybrid real protocol and in the ideal process. Thus, both the $\mathcal{F}$-hybrid real protocol and the ideal process continue correctly in this MAC phase.

Proof of Theorem 1 Let $\mathcal{A}$ be an adversary that interacts with participants running in the $\mathcal{F}$-hybrid real protocol. Then, we construct a simulator $\mathcal{S}$, such that no environment $\mathcal{Z}$ can distinguish whether it is interacting with $\mathcal{A}$ and participants running in the $\mathcal{F}$-hybrid real protocol, or with $\mathcal{S}$ and participants that interact with $\mathcal{F}_{\mathrm{C} 2 \mathrm{C}-\text { GAKE }}$ in the ideal process. The simulator $\mathcal{S}$ simulates the behavior of the adversary $\mathcal{A}$, and proceeds as follows:

- Common items to phases:

- Any input from $\mathcal{Z}$ is forwarded to $\mathcal{A}$, and any output of $\mathcal{A}$ is copied to the output of
$\mathcal{S}$ (to be read by $\mathcal{Z}$ ).

- Whenever $\mathcal{A}$ corrupts a participant, $\mathcal{S}$ corrupts the same participant in the ideal process, and provides $\mathcal{A}$ with the internal state of the corrupted participant.

- UCPAKE phase between $C$ and $S$ :

- If $\mathcal{A}$ makes a value (TestPwd, sid, $C, p w_{C}^{\prime \prime}$ ) for $\mathcal{F}_{\text {pwKE }}$, then $\mathcal{S}$ makes the same value to $\mathcal{F}_{\mathrm{C} 2 \mathrm{C}-\mathrm{GAKE}}$ and provides $\mathcal{A}$ with the reply related to the state of the session (namely, compromised or interrupted).

- When an uncorrupted client $C$ is activated with some input, $\mathcal{S}$ obtains this value from $\mathcal{F}_{\mathrm{C} 2 \mathrm{C}-\mathrm{GAKE}}$. Then, $\mathcal{S}$ simulates for $\mathcal{A}$ the expected interaction with $\mathcal{F}_{\text {pwKE }}$; that is, $\mathcal{S}$ sends to $\mathcal{A}$ (in the name of $\mathcal{F}_{\text {pwKE }}$ ) the message (NewSession, sid, $C, S$, initiator), obtains (NewKey, sid, $C, s k_{C}$ ) from $\mathcal{A}$, and forwards this value to $\mathcal{F}_{\mathrm{C} 2 \mathrm{C}-\mathrm{GAKE}}$. If $\left(C, S, p w_{C}\right)$ is compromised, $\mathcal{S}$ forwards $\left(\right.$ sid, $\left.s k_{C}\right)$ to $C$.

- Whenever a corrupted client $C$ sends a message (NewSession, sid, $C, S$, initiator) to $\mathcal{F}_{\text {pwKE }}, \mathcal{S}$ responds with this value to $\mathcal{A}$ in the name of $\mathcal{F}_{\text {pwKE }}$, obtains a random key (NewKey, sid, $C, s k_{C}$ ) from $\mathcal{A}$, sends this value to $\mathcal{F}_{\mathrm{C} 2 \mathrm{C}-\mathrm{GAKE}}$, and forwards $\left(\right.$ sid, $\left.s k_{C}\right)$ to $C$.

- When a server $S$ is activated with some input, $\mathcal{S}$ obtains this value from $\mathcal{F}_{\mathrm{C} 2 \mathrm{C}-\mathrm{GAKE}}$. Then, $\mathcal{S}$ simulates for $\mathcal{A}$ the expected interaction with $\mathcal{F}_{\text {pwKE}}$; that is, $\mathcal{S}$ sends to $\mathcal{A}$ (in the name of $\mathcal{F}_{\text {pwKE }}$ ) the message (NewSession, sid, $S, C$, responder), obtains (NewKey, sid, $S, s k_{C}$ ) from $\mathcal{A}$, and forwards this value to $\mathcal{F}_{\mathrm{C} 2 \mathrm{C}-\mathrm{GAKE}}$.

- SIGDH phase between $S_{1}$ and $S_{2}$ :

- When a server $S_{1}$ (resp. $S_{2}$ ) is activated with some input, $\mathcal{S}$ obtains this value from $\mathcal{F}_{\mathrm{C} 2 \mathrm{C}-\mathrm{GAKE}}$. Then, $\mathcal{S}$ simulates for $\mathcal{A}$ the expected interaction with $\mathcal{F}_{\mathrm{KE}}$; that is, $\mathcal{S}$ sends to $\mathcal{A}$ (in the name of $\mathcal{F}_{\mathrm{KE}}$ ) the message (NewSession, sid, $S_{1}, S_{2}$, initiator) (resp. (NewSession, sid, $S_{2}, S_{1}$, responder)), obtains $\left(s i d, s k_{S}\right)$ from $\mathcal{A}$, and forwards (NewKey, sid, $S_{1}, s k_{S}$ ) (resp. (NewKey, sid, $\left.S_{2}, s k_{S}\right)$ ) to $\mathcal{F}_{\mathrm{C} 2 \mathrm{C}-\mathrm{GAKE}}$.

- MAC phase from $C$ to $S$ :

- When the following occurs, the protocol halts:

* $C$ and/or $S$ do not have corresponding MAC keys.

* $C$ and $S$ have different MAC keys.

- When an uncorrupted client $C$ is ac- 
tivated with some input, $\mathcal{S}$ obtains this value from $\mathcal{F}_{\mathrm{C} 2 \mathrm{C}-\mathrm{GAKE}}$. Then, $\mathcal{S}$ simulates for $\mathcal{A}$ the expected interaction with $\mathcal{F}_{\text {AUTH }}$; that is, $\mathcal{S}$ sends to $\mathcal{A}$ (in the name of $\mathcal{F}_{\text {AUTH }}$ ) the message (sent, sid, $C, S, g^{r}$ ), obtains (send, sid, $S^{\prime}, g^{r^{\prime}}$ ) from $\mathcal{A}$, and forwards this value to $\mathcal{F}_{\mathrm{C} 2 \mathrm{C}-\mathrm{GAKE}}$.

- Whenever a corrupted client $C$ sends a message (send, sid, $S, g^{r}$ ) to $\mathcal{F}_{\text {AUTH }}, \mathcal{S}$ responds with (sent, sid, $C, S, g^{r}$ ) to $\mathcal{A}$ in the name of $\mathcal{F}_{\mathrm{AUTH}}$, obtains (send, sid, $S^{\prime}$, $g^{r^{\prime}}$ ) from $\mathcal{A}$, sends (send, sid, $S^{\prime}, g^{r^{\prime}}$ ) to $\mathcal{F}_{\mathrm{C} 2 \mathrm{C}-\mathrm{GAKE}}$, and forwards (sent, sid, $C, g^{r^{\prime}}$ ) to the unintended server $S^{\prime}$ (if $S^{\prime} \neq S$ ) or the partner server $S$ (if $S^{\prime}=S$ ).

- MAC phase from $S_{1}$ to $S_{2}$ (resp. from $S_{2}$ to $\left.S_{1}\right)$ :

- When the following occurs, the protocol halts:

* $S_{1}$ and/or $S_{2}$ do not have corresponding MAC keys.

* $S_{1}$ and $S_{2}$ have different MAC keys.

- When a server $S_{1}$ (resp. $S_{2}$ ) is activated with some input, $\mathcal{S}$ obtains this value from

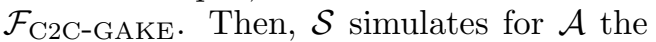
expected interaction with $\mathcal{F}_{\text {AUTH }}$; that is, $\mathcal{S}$ sends to $\mathcal{A}$ (in the name of $\mathcal{F}_{\mathrm{AUTH}}$ ) the message (sent, sid, $S_{1}, S_{2}, g^{r}$ ) (resp. (sent, sid, $\left.S_{2}, S_{1}, g^{r}\right)$ ), obtains (send, sid, $S_{2}^{\prime}, g^{r^{\prime}}$ ) (resp. (send, sid, $\left.S_{1}^{\prime}, g^{r^{\prime}}\right)$ ) from $\mathcal{A}$, and forwards this value to $\mathcal{F}_{\mathrm{C} 2 \mathrm{C}-\text { GAKE }}$.

- MAC phase from $S$ to $C$ :

- When the following occurs, the protocol halts:

* $S$ and/or $C$ do not have corresponding MAC keys.

* $S$ and $C$ have different MAC keys.

- When a server $S$ is activated with some input, $\mathcal{S}$ obtains this value from

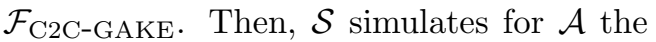
expected interaction with $\mathcal{F}_{\text {AUTH }}$; that is, $\mathcal{S}$ sends to $\mathcal{A}$ (in the name of $\mathcal{F}_{\mathrm{AUTH}}$ ) the message (sent, sid, $S, C, g^{r}$ ), obtains (send, sid, $C^{\prime}, g^{r^{\prime}}$ ) from $\mathcal{A}$, and forwards this value to $\mathcal{F}_{\mathrm{C} 2 \mathrm{C}-\mathrm{GAKE}}$.

- MAC phase from $U_{i}$ to $U_{j}$

- Whenever $\mathcal{A}$ passively corrupts a server, $\mathcal{S}$ passively corrupts the same server in the ideal process, and provides $\mathcal{A}$ with the internal state $g^{r_{1}}$ or $g^{r_{2}}$ of the corrupted server.

- $\mathcal{S}$ constructs a distinguisher $\mathcal{D}$ that breaks the DDH assumption. The input to
$\mathcal{D}$ is denoted by $(\alpha, \beta, \gamma)$ that is related to $Q_{0}$ or $Q_{1}$ each with probability $1 / 2$ according to a random bit $b \in\{0,1\}$, where $Q_{0}=$ $\left(g^{r_{1}}, g^{r_{2}}, g^{r_{1} r_{2}}\right)$ and $Q_{1}=\left(g^{r_{1}}, g^{r_{2}}, g^{r_{3}}\right)$, and $r_{3}$ is random. Then, $\mathcal{D}$ outputs the guess bit $b^{\prime}$ for $b$, and $\mathcal{S}$ also outputs the bit $b^{\prime}$.

We here consider whether the above simulation is valid. We partly adopt abbreviations for simplicity, hereafter.

- If $A$ and $B$ are uncorrupted:

- UCPAKE phase between $C$ and $S$ :

* In the $\mathcal{F}$-hybrid real protocol, $C$ and $S$ send NewSession to $\mathcal{F}_{\text {pwKE. If }}$ $\left(C, S, p w_{C}\right)$ is not compromised, then $C$ and $S$ obtain random session keys from $\mathcal{F}_{\text {pwKe }}$, respectively. In this case, $C$ and $S$ usually share the same session key, but may share different session keys by the definition of $\mathcal{F}_{\text {pwKE }}$, since the password guessing attack by the adversary has failed or one of them has inputted the incorrect password by mistake. Otherwise, $C$ and $S$ obtain random session keys from $\mathcal{S}$ by $\mathcal{F}_{\text {pwKe }}$, respectively. In this case, $C$ and $S$ share the same session key by the definition of $\mathcal{F}_{\text {pwKE }}$.

* In the ideal process, $C$ and $S$ are activated with some input, $\mathcal{F}_{\mathrm{C} 2 \mathrm{C}-\mathrm{GAKE}}$ sends this value to $\mathcal{S}$, and $\mathcal{S}$ simulates for $\mathcal{A}$ the expected interaction with $\mathcal{F}_{\text {pwKE }}$, respectively. Then, $\mathcal{S}$ obtains random session keys from $\mathcal{A}$ in the name of $\mathcal{F}_{\text {pwKE }}$, and sends these keys to $\mathcal{F}_{\mathrm{C} 2 \mathrm{C}-\mathrm{GAKE}}$ respectively. In this case, if $\left(C, S, p w_{C}\right)$ is not compromised and these keys received by $\mathcal{F}_{\mathrm{C} 2 \mathrm{C}-\mathrm{GAKE}}$ are the same for $C$ and $S$, that is, if the passwords inputted by them are the same, then $\mathcal{F}_{\mathrm{C} 2 \mathrm{C}-\mathrm{GAKE}}$ chooses a random session key, and sends this key to $C$ and $S$ respectively. If $\left(C, S, p w_{C}\right)$ is not compromised and these keys received by $\mathcal{F}_{\mathrm{C} 2 \mathrm{C}-\text { GAKE }}$ are different for $C$ and $S$, that is, if the passwords inputted by them are different or the failed password guessing attack is carried out by the adversary, $\mathcal{F}_{\mathrm{C} 2 \mathrm{C}-\text { GAKE }}$ forwards the respective keys from $\mathcal{S}$ to $C$ and $S$. If $\left(C, S, p w_{C}\right)$ is compromised, $\mathcal{F}_{\mathrm{C} 2 \mathrm{C}-\mathrm{GAKE}}$ sends $C$ and $S$ the keys from $\mathcal{S}$ respectively. In this case, $C$ and $S$ hold the same key generated by $\mathcal{A}$.

* If the keys of $C$ and $S$ are the same in the $\mathcal{F}$-hybrid real protocol, they are the 
same in the ideal process; otherwise they are different.

Remark 2: If the adversary makes a successful password guess (that is, a session is compromised), then the adversary can determine the session key for compromised sessions, and the participant is provided with this session key. If the adversary makes an incorrect password guess (that is, a session is interrupted), then the participant is provided with a randomly chosen session key. The above shows the following: When the adversary is successful in the password guess, which corresponds to the success of the on-line dictionary attack, the adversary then learns a session key. Meanwhile, when the adversary fails in the password guess, the participant receives a different session key from the key received by its partner, which is the same as this participant receives the error message. This is because the participant learns failure of the password guess by the adversary, and it is impossible for the adversary to carry out the off-line dictionary attack. This implies that the success probability of the password guess by the adversary is equivalent to $(1 /|\mathcal{D}|)$ in the ideal process, where $|\mathcal{D}|$ denotes the size of the dictionary $\mathcal{D}$. On the other hand, it is guaranteed that the success probability of the password guess by the adversary also becomes equivalent to $(1 /|\mathcal{D}|)$ in the $\mathcal{F}$-hybrid real proto$\mathrm{col}$, since the above functionality is securely realized. Consequently, it is shown that the protocol that securely realizes the proposed functionality is resistant to the off-line dictionary attack.

- SIGDH phase between $S_{1}$ and $S_{2}$ :

* In the $\mathcal{F}$-hybrid real protocol, $S_{1}$ and $S_{2}$ send NewSession to $\mathcal{F}_{\mathrm{KE}}$, and obtain the keys from $\mathcal{F}_{\mathrm{KE}}$, respectively. In this case, $S_{1}$ and $S_{2}$ share the same session key by the definition of $\mathcal{F}_{\mathrm{KE}}$.

* In the ideal process, $S_{1}$ and $S_{2}$ are activated with some input, $\mathcal{F}_{\mathrm{C} 2 \mathrm{C}-\mathrm{GAKE}}$ sends this value to $\mathcal{S}$, and $\mathcal{S}$ simulates for $\mathcal{A}$ the expected interaction with $\mathcal{F}_{\mathrm{KE}}$, respectively. Then, $\mathcal{S}$ obtains the key from $\mathcal{A}$ in the name of $\mathcal{F}_{\mathrm{KE}}$, and sends this key to $\mathcal{F}_{\mathrm{C} 2 \mathrm{C} \text {-GAKE }}$ respectively. In this case, $\mathcal{F}_{\mathrm{C} 2 \mathrm{C}-\mathrm{GAKE}}$ chooses a random session key, and sends this key to $S_{1}$ and $S_{2}$ respectively. Hence, the keys of $S_{1}$ and
$S_{2}$ are also the same, since they are the same in the $\mathcal{F}$-hybrid real protocol.

- MAC phase from $U_{i}$ to $U_{j}$ :

* In the $\mathcal{F}$-hybrid real protocol, $U_{i}$ sends send to $\mathcal{F}_{\text {AUTH }}$, and $U_{j}$ obtains sent from $\mathcal{F}_{\text {AUTH. }}$ In this case, $U_{i}$ and $U_{j}$ hold the same message by the definition of $\mathcal{F}_{\text {AUTH }}$.

* In the ideal process, $U_{i}$ is activated with some input, $\mathcal{F}_{\mathrm{C} 2 \mathrm{C}-\mathrm{GAKE}}$ sends this value to $\mathcal{S}$, and $\mathcal{S}$ simulates for $\mathcal{A}$ the expected interaction with $\mathcal{F}_{\text {AUTH }}$. Then, $\mathcal{S}$ obtains send from $\mathcal{A}$ in the name of $\mathcal{F}_{\mathrm{AUTH}}$, and $\mathcal{F}_{\mathrm{C} 2 \mathrm{C}-\mathrm{GAKE}}$ sends $U_{j}$ the message from $U_{i}$. In this case, the messages of $U_{i}$ and $U_{j}$ are also the same, since they are the same in the $\mathcal{F}$-hybrid real protocol.

* If $C$ and $S$ share different MAC keys in the UCPAKE phase, then both the $\mathcal{F}$ hybrid real protocol and the ideal process halt in the MAC phase from $C$ to $S$. This is because MAC verification meets with failure in this MAC phase.

- If $A$ is corrupted in the UCPAKE phase between $A$ and $S_{1}$ :

- UCPAKE phase between $A$ and $S_{1}$ :

* In the $\mathcal{F}$-hybrid real protocol, $A$ and $S_{1}$ send NewSession to $\mathcal{F}_{\text {pwKE }}$, and obtain random session keys from $\mathcal{S}$ by $\mathcal{F}_{\text {pwKE }}$, respectively. In this case, $A$ and $S_{1}$ share the same session key by the definition of $\mathcal{F}_{\text {pwKE. }}$.

* In the ideal process, $A$ and $S_{1}$ are activated with some input, $\mathcal{F}_{\mathrm{C} 2 \mathrm{C}-\mathrm{GAKE}}$ sends this value to $\mathcal{S}$, and $\mathcal{S}$ simulates for $\mathcal{A}$ the expected interaction with $\mathcal{F}_{\text {pwKE }}$, respectively. Then, $\mathcal{S}$ obtains the random session keys from $\mathcal{A}$ in the name of $\mathcal{F}_{\text {pwKE }}$, and $\mathcal{F}_{\mathrm{C} 2 \mathrm{C}-\mathrm{GAKE}}$ sends $A$ and $S_{1}$ the keys from $\mathcal{S}$ respectively. In this case, $A$ and $S_{1}$ hold the same key generated by $\mathcal{A}$. Hence, the keys of $A$ and $S_{1}$ are also the same, since they are the same in the $\mathcal{F}$ hybrid real protocol.

- MAC phase from $A$ to $S_{1}$ :

* In the $\mathcal{F}$-hybrid real protocol, if $S_{1}^{\prime} \neq$ $S_{1}, A$ sends $g^{r_{1}}$ to $\mathcal{F}_{\text {AUTH }}$, and the unintended server $S_{1}^{\prime}$ obtains $g^{r_{1}^{\prime}}$ from $\mathcal{S}$ by $\mathcal{F}_{\text {AUTH }}$. In this case, $S_{1}$ cannot receive the message related to the final session key by the definition of $\mathcal{F}_{\text {AUTH. }}$. On the other hand, if $S_{1}^{\prime}=S_{1}, A$ sends $g^{r_{1}}$ to $\mathcal{F}_{\mathrm{AUTH}}$, and the partner server $S_{1}$ ob- 
tains $g^{r_{1}^{\prime}}$ from $\mathcal{S}$ by $\mathcal{F}_{\text {AUTH }}$. In this case, $S_{1}$ receives the message the adversary has altered by the definition of $\mathcal{F}_{\text {AUTH }}$.

* In the ideal process, $A$ is activated with some input, $\mathcal{F}_{\mathrm{C} 2 \mathrm{C}-\mathrm{GAKE}}$ sends this value to $\mathcal{S}$, and $\mathcal{S}$ simulates for $\mathcal{A}$ the expected interaction with $\mathcal{F}_{\text {AUTH }}$. Then, $\mathcal{S}$ obtains $g^{r_{1}^{\prime}}$ from $\mathcal{A}$ in the name of $\mathcal{F}_{\mathrm{AUTH}}$, and $\mathcal{F}_{\mathrm{C} 2 \mathrm{C}-\mathrm{GAKE}}$ sends $S_{1}^{\prime}$ the message from $\mathcal{S}$ if $S_{1}^{\prime} \neq S_{1}$. In this case, $S_{1}$ cannot receive the message related to the final session key. On the other hand, $\mathcal{F}_{\mathrm{C} 2 \mathrm{C}-\mathrm{GAKE}}$ sends $S_{1}$ the message from $\mathcal{S}$ if $S_{1}^{\prime}=S_{1}$.

* If $S_{1}^{\prime} \neq S_{1}$, both the $\mathcal{F}$-hybrid real protocol and the ideal process halt in this MAC phase, since $S_{1}$ cannot receive the message related to the final session key in this phase. On the other hand, if $S_{1}^{\prime}=S_{1}$, both the $\mathcal{F}$-hybrid real protocol and the ideal process continue correctly in this MAC phase, since $S_{1}$ receives the message although the adversary has altered it.

- If $A$ is corrupted in the MAC phase from $A$ to $S_{1}$ :

- MAC phase from $A$ to $S_{1}$ :

* In the $\mathcal{F}$-hybrid real protocol, if $S_{1}^{\prime} \neq$ $S_{1}, A$ sends $g^{r_{1}}$ to $\mathcal{F}_{\mathrm{AUTH}}$, and the unintended server $S_{1}^{\prime}$ obtains $g^{r_{1}^{\prime}}$ from $\mathcal{S}$ by $\mathcal{F}_{\text {AUTH }}$. In this case, $S_{1}$ cannot receive the message related to the final session key by the definition of $\mathcal{F}_{\mathrm{AUTH}}$. On the other hand, if $S_{1}^{\prime}=S_{1}, A$ sends $g^{r_{1}}$ to $\mathcal{F}_{\text {AUTH }}$, and the partner server $S_{1}$ obtains $g^{r_{1}^{\prime}}$ from $\mathcal{S}$ by $\mathcal{F}_{\text {AUTH }}$. In this case, $S_{1}$ receives the message the adversary has altered by the definition of $\mathcal{F}_{\text {AUTH }}$.

* In the ideal process, $A$ is activated with some input, $\mathcal{F}_{\mathrm{C} 2 \mathrm{C}-\mathrm{GAKE}}$ sends this value to $\mathcal{S}$, and $\mathcal{S}$ simulates for $\mathcal{A}$ the expected interaction with $\mathcal{F}_{\text {AUTH }}$. Then, $\mathcal{S}$ obtains $g^{r_{1}^{\prime}}$ from $\mathcal{A}$ in the name of $\mathcal{F}_{\text {AUTH }}$, and $\mathcal{F}_{\mathrm{C} 2 \mathrm{C}-\mathrm{GAKE}}$ sends $S_{1}^{\prime}$ the message from $\mathcal{S}$ if $S_{1}^{\prime} \neq S_{1}$. In this case, $S_{1}$ cannot receive the message related to the final session key. On the other hand, $\mathcal{F}_{\mathrm{C} 2 \mathrm{C}-\mathrm{GAKE}}$ sends $S_{1}$ the message from $\mathcal{S}$ if $S_{1}^{\prime}=S_{1}$.

* If $S_{1}^{\prime} \neq S_{1}$, both the $\mathcal{F}$-hybrid real protocol and the ideal process halt in this MAC phase, since $S_{1}$ cannot receive the message related to the final session key in this phase. On the other hand, if $S_{1}^{\prime}=S_{1}$, both the $\mathcal{F}$-hybrid real protocol and the ideal process continue correctly in this MAC phase, since $S_{1}$ receives the message although the adversary has altered it.

- If $B$ is corrupted in the UCPAKE phase between $B$ and $S_{2}$ :

- UCPAKE phase between $B$ and $S_{2}$ :

* In the $\mathcal{F}$-hybrid real protocol, $B$ and $S_{2}$ send NewSession to $\mathcal{F}_{\text {pwKE }}$, and obtain random session keys from $\mathcal{S}$ by $\mathcal{F}_{\text {pwKE }}$, respectively. In this case, $B$ and $S_{2}$ share the same session key by the definition of $\mathcal{F}_{\text {pwKE. }}$.

* In the ideal process, $B$ and $S_{2}$ are activated with some input, $\mathcal{F}_{\mathrm{C} 2 \mathrm{C}-\mathrm{GAKE}}$ sends this value to $\mathcal{S}$, and $\mathcal{S}$ simulates for $\mathcal{A}$ the expected interaction with $\mathcal{F}_{\text {pwKE }}$, respectively. Then, $\mathcal{S}$ obtains the random session keys from $\mathcal{A}$ in the name of $\mathcal{F}_{\text {pwKE }}$, and $\mathcal{F}_{\mathrm{C} 2 \mathrm{C}-\mathrm{GAKE}}$ sends $B$ and $S_{2}$ the keys from $\mathcal{S}$ respectively. In this case, $B$ and $S_{2}$ hold the same key generated by $\mathcal{A}$. Hence, the keys of $B$ and $S_{2}$ are also the same, since they are the same in the $\mathcal{F}$ hybrid real protocol.

- MAC phase from $B$ to $S_{2}$ :

* In the $\mathcal{F}$-hybrid real protocol, if $S_{2}^{\prime} \neq$ $S_{2}, B$ sends $g^{r_{2}}$ to $\mathcal{F}_{\mathrm{AUTH}}$, and the unintended server $S_{2}^{\prime}$ obtains $g^{r_{2}^{\prime}}$ from $\mathcal{S}$ by $\mathcal{F}_{\text {AUTH. }}$. In this case, $S_{2}$ cannot receive the message related to the final session key by the definition of $\mathcal{F}_{\text {AUTH }}$. On the other hand, if $S_{2}^{\prime}=S_{2}, B$ sends $g^{r_{2}}$ to $\mathcal{F}_{\text {AUTH }}$, and the partner server $S_{2}$ obtains $g^{r_{2}^{\prime}}$ from $\mathcal{S}$ by $\mathcal{F}_{\text {AUTH }}$. In this case, $S_{2}$ receives the message the adversary has altered by the definition of $\mathcal{F}_{\text {AUTH }}$.

* In the ideal process, $B$ is activated with some input, $\mathcal{F}_{\mathrm{C} 2 \mathrm{C}-\mathrm{GAKE}}$ sends this value to $\mathcal{S}$, and $\mathcal{S}$ simulates for $\mathcal{A}$ the expected interaction with $\mathcal{F}_{\text {AUTH }}$. Then, $\mathcal{S}$ obtains $g^{r_{2}^{\prime}}$ from $\mathcal{A}$ in the name of $\mathcal{F}_{\mathrm{AUTH}}$, and $\mathcal{F}_{\mathrm{C} 2 \mathrm{C}-\mathrm{GAKE}}$ sends $S_{2}^{\prime}$ the message from $\mathcal{S}$ if $S_{2}^{\prime} \neq S_{2}$. In this case, $S_{2}$ cannot receive the message related to the final session key. On the other hand, $\mathcal{F}_{\mathrm{C} 2 \mathrm{C}-\text { GAKE }}$ sends $S_{2}$ the message from $\mathcal{S}$ if $S_{2}^{\prime}=S_{2}$.

* If $S_{2}^{\prime} \neq S_{2}$, both the $\mathcal{F}$-hybrid real protocol and the ideal process halt in this MAC phase, since $S_{2}$ cannot receive the message related to the final session key 
in this phase. On the other hand, if $S_{2}^{\prime}=S_{2}$, both the $\mathcal{F}$-hybrid real protocol and the ideal process continue correctly in this MAC phase, since $S_{2}$ receives the message although the adversary has altered it.

- If $B$ is corrupted in the MAC phase from $B$ to $S_{2}$ :

- MAC phase from $B$ to $S_{2}$ :

* In the $\mathcal{F}$-hybrid real protocol, if $S_{2}^{\prime} \neq$ $S_{2}, B$ sends $g^{r_{2}}$ to $\mathcal{F}_{\mathrm{AUTH}}$, and the unintended server $S_{2}^{\prime}$ obtains $g^{r_{2}^{\prime}}$ from $\mathcal{S}$ by $\mathcal{F}_{\text {AUTH }}$. In this case, $S_{2}$ cannot receive the message related to the final session key by the definition of $\mathcal{F}_{\text {AUTH }}$. On the other hand, if $S_{2}^{\prime}=S_{2}, B$ sends $g^{r_{2}}$ to $\mathcal{F}_{\text {AUTH }}$, and the partner server $S_{2}$ obtains $g^{r_{2}^{\prime}}$ from $\mathcal{S}$ by $\mathcal{F}_{\text {AUTH }}$. In this case, $S_{2}$ receives the message the adversary has altered by the definition of $\mathcal{F}_{\text {AUTH }}$.

* In the ideal process, $B$ is activated with some input, $\mathcal{F}_{\mathrm{C} 2 \mathrm{C}-\mathrm{GAKE}}$ sends this value to $\mathcal{S}$, and $\mathcal{S}$ simulates for $\mathcal{A}$ the expected interaction with $\mathcal{F}_{\text {AUTH }}$. Then, $\mathcal{S}$ obtains $g^{r_{2}^{\prime}}$ from $\mathcal{A}$ in the name of $\mathcal{F}_{\mathrm{AUTH}}$, and $\mathcal{F}_{\mathrm{C} 2 \mathrm{C}-\mathrm{GAKE}}$ sends $S_{2}^{\prime}$ the message from $\mathcal{S}$ if $S_{2}^{\prime} \neq S_{2}$. In this case, $S_{2}$ cannot receive the message related to the final session key. On the other hand, $\mathcal{F}_{\mathrm{C} 2 \mathrm{C}-\mathrm{GAKE}}$ sends $S_{2}$ the message from $\mathcal{S}$ if $S_{2}^{\prime}=S_{2}$.

* If $S_{2}^{\prime} \neq S_{2}$, both the $\mathcal{F}$-hybrid real protocol and the ideal process halt in this MAC phase, since $S_{2}$ cannot receive the message related to the final session key in this phase. On the other hand, if $S_{2}^{\prime}=S_{2}$, both the $\mathcal{F}$-hybrid real protocol and the ideal process continue correctly in this MAC phase, since $S_{2}$ receives the message although the adversary has altered it.

- If $S_{1}$ and $S_{2}$ are passively corrupted in the MAC phase from $U_{i}$ to $U_{j}$ :

- MAC phase from $U_{i}$ to $U_{j}$

* In the $\mathcal{F}$-hybrid real protocol, when $\mathcal{A}$ passively corrupts $S_{1}$ and $S_{2}$, it obtains the internal states $g^{r_{1}}$ and $g^{r_{2}}$ of them. $\mathcal{A}$ is given $(\alpha, \beta, \gamma)$ that is related to $Q_{0}$ or $Q_{1}$ each with probability $1 / 2$ according to a random bit $b \in\{0,1\}$. Then, $\mathcal{A}$ guesses the value $b$, and outputs the guess $b^{\prime}$. In this case, $\mathcal{A}$ can correctly guess the bit $b$ only with negligible advantage due to the DDH assumption.
* In the ideal process, $\mathcal{S}$ is given $(\alpha, \beta, \gamma)$ that is related to $Q_{0}$ or $Q_{1}$ each with probability $1 / 2$ according to a random bit $b \in\{0,1\}$. Then, $\mathcal{S}$ outputs the guess bit $b^{\prime}$ received from the distinguisher $\mathcal{D}$. In this case, $\mathcal{D}$ can correctly guess the bit $b$ only with negligible advantage due to the DDH assumption. Therefore, $\mathcal{S}$ can also correctly output the bit $b$ only with negligible advantage, regardless of $\mathcal{S}$ 's ability.

* Both in the $\mathcal{F}$-hybrid real protocol and the ideal process, the guess bit $b^{\prime}$ can correctly be outputted only with negligible advantage due to the DDH assumption.

It is easy to see that this simulation is perfect. The view of $\mathcal{Z}$ in an interaction with $\mathcal{S}$ and participants in the ideal process, is distributed identically to its view in an interaction with $\mathcal{A}$ and participants running in the $\mathcal{F}$-hybrid real protocol.

\section{Conclusion}

In this paper, we defined the client-to-client general authenticated key exchange functionality $\mathcal{F}_{\mathrm{C} 2 \mathrm{C}-\mathrm{GAKE}}$, which is composed of the Setup phase and MAC phase such as existing functionalities. This functionality is defined such that inconsistency does not occur between respective phases. We also proposed the clientto-client general authenticated key exchange protocol C2C-GAKE as a general form of the client-to-client model, which is composed of the Setup and MAC protocols, and the client-toclient hybrid authenticated key exchange protocol C2C-HAKE as an example protocol of C2CGAKE, which is composed of the UC-secure 2-party password-based key exchange protocol UCPAKE, the UC-secure authenticated DH key exchange protocol via digital signatures SIGDH, and the message authentication that realizes the secure MAC algorithm in the UC framework. We proved that C2C-HAKE securely realizes the functionality $\mathcal{F}_{\mathrm{C} 2 \mathrm{C}-\mathrm{GAKE}}$. C2C-HAKE is the first client-to-client hybrid authenticated key exchange protocol that is secure in the UC framework with a security-preserving composition property.

\section{References}

1) Byun, J., Jeong, I., Lee, D. and Park, C.: Password-Authenticated Key Exchange between Clients with Different Passwords, 
Information and Communications Security (ICICS 2002), LNCS, Vol.2513, pp.134-146, Singapore, Springer-Verlag (2002).

2) Chen, L.: A Weakness of the PasswordAuthenticated Key Agreement between Clients with Different Passwords Scheme, Circulated for consideration at the 27th SC27/WG2 meeting, Paris, France (2003).

3) Wang, S., Wang, J. and Xu, M.: Weaknesses of a Password-Authenticated Key Exchange Protocol between Clients with Different Passwords, Applied Cryptography and Network Security - ACNS 2004, LNCS, Vol.3089, pp.414-425, Yellow Mountain, China, SpringerVerlag (2004).

4) Kim, J., Kim, S., Kwak, J. and Won, D.: Cryptanalysis and Improvement of Password Authenticated Key Exchange Scheme between Clients with Different Passwords, Computational Science and Its Applications - ICCSA 2004, LNCS, Vol.3043, pp.895-902, Perugia, Italy, Springer-Verlag (2004).

5) Phan, R.-W. and Goi, B.-M.: Cryptanalysis of an Improved Client-to-Client PasswordAuthenticated Key Exchange (C2C-PAKE) Scheme, Applied Cryptography and Network Security - ACNS 2005, LNCS, Vol.3531, pp.33-39, New York, NY, USA, SpringerVerlag (2005).

6) Byun, J., Lee, D. and Lim, J.: Efficient and Provably Secure Client-to-Client PasswordBased Key Exchange Protocol, Frontiers of $W W W$ Research and Development - APWeb 2006, LNCS, Vol.3841, pp.830-836, Harbin, China, Springer-Verlag (2006).

7) Yin, Y. and Bao, L.: Secure Cross-Realm C2C-PAKE Protocol, Information Security and Privacy (ACISP 2006), LNCS, Vol.4058, pp.395-406, Melbourne, Australia, SpringerVerlag (2006).

8) Canetti, R.: Universally Composable Security: A New Paradigm for Cryptographic Protocols, Proc. 42nd Symposium on Foundations of Computer Science (FOCS 2001), Las Vegas, NV, USA (2001).

9) Canetti, R. and Krawczyk, H.: Universally Composable Notions of Key Exchange and Secure Channels, Advances in Cryptology EUROCRYPT 2002, LNCS, Vol.2332, pp.337351, Amsterdam, the Netherlands, SpringerVerlag (2002).

10) Canetti, R.: Universally Composable Signature, Certification, and Authentication, Proc. 17th IEEE Computer Security Foundations Workshop (CSFW 2004), Pacific Grove, CA, USA (2004).

11) Canetti, R., Halevi, S., Katz, J., Lindell,
Y. and MacKenzie, P.: Universally Composable Password-Based Key Exchange, Advances in Cryptology - EUROCRYPT 2005, LNCS, Vol.3494, pp.404-421, Aarhus, Denmark, Springer-Verlag (2005).

12) Bellare, M. and Rogaway, P.: Entity Authentication and Key Distribution, Advances in Cryptology - CRYPTO'93, LNCS, Vol.773, pp.232-249, Santa Barbara, CA, USA, Springer-Verlag (1993).

13) Bellare, M. and Rogaway, P.: Provably Secure Session Key Distribution - The Three Party Case, Proc. 27th Annual ACM Symposium on Theory of Computing, Philadelphia, PA, USA, pp.57-66, ACM Press (1996).

14) Bellare, M., Canetti, R. and Krawczyk, H.: A Modular Approach to the Design and Analysis of Authentication and Key Exchange Protocols, Proc.30th ACM Symposium on the Theory of Computing, Dallas, TX, USA, pp.419-428 (1998).

15) Bellovin, S. and Merritt, M.: Encrypted Key Exchange: Password-Based Protocols Secure Against Dictionary Attacks, Proc. IEEE Computer Society Symposium on Research in Security and Privacy, Oakland, CA, USA, pp.72-84 (1992).

16) Halevi, S. and Krawczyk, H.: Public-key cryptography and password protocols, Proc. 5th ACM Conference on Computer and Communications Security, San Francisco, CA, USA, pp.122-131 (1998).

17) Halevi, S. and Krawczyk, H.: Public-key cryptography and password protocols, ACM Trans. on Information and System Security, Vol.2, No.3, pp.230-268 (1999).

18) Bellare, M., Pointcheval, D. and Rogaway, P.: Authenticated Key Exchange Secure Against Dictionary Attacks, Advances in Cryptology EUROCRYPT 2000, LNCS, Vol.1807, pp.139155, Bruges, Belgium, Springer-Verlag (2000).

19) Boyko, V., MacKenzie, P. and Patel, S.: Provably Secure Password Authenticated Key Exchange Using Diffie-Hellman, Advances in Cryptology - EUROCRYPT 2000, LNCS, Vol.1807, pp.156-171, Bruges, Belgium, Springer-Verlag (2000).

20) Boyarsky, M.: Public-Key Cryptography and Password Protocols: The Multi-User Case, Proc. 6th ACM Conference on Computer and Communications Security, Singapore, pp.6372 (1999).

21) MacKenzie, P. and Swaminathan, R.: Secure Network Authentication with Password Identification, IEEE P1363.2 (1999).

22) MacKenzie, P., Patel, S. and Swaminathan, R.: Password-Authenticated Key Exchange 
based on RSA, Advances in Cryptology ASIACRYPT 2000, LNCS, Vol.1976, pp.599613, Kyoto, Japan, Springer-Verlag (2000).

23) Goldreich, O. and Lindell, Y.: Session-Key Generation using Human Passwords Only, Advances in Cryptology - CRYPTO 2001, LNCS, Vol.2139, pp.408-432, Santa Barbara, CA, USA, Springer-Verlag (2001).

24) Katz, J., Ostrovsky, R. and Yung, M.: Efficient Password-Authenticated Key Exchange Using Human-Memorable Passwords, Advances in Cryptology - EUROCRYPT 2001, LNCS, Vol.2045, pp.475-494, Innsbruck, Austria, Springer-Verlag (2001).

25) MacKenzie, P.: The PAK suite: Protocols for Password-Authenticated Key Exchange, IEEE P1363.2 (2002).

26) Kobara, K. and Imai, H.: Pretty-Simple Password-Authenticated Key-Exchange Protocol Proven to be Secure in the Standard Model, IEICE Trans. Fundamentals, Vol.E85-A, No.10, pp.2229-2237 (2002).

27) Gennaro, R. and Lindell, Y.: A Framework for Password-Based Authenticated Key Exchange, Advances in Cryptology - EUROCRYPT 2003, LNCS, Vol.2656, pp.524-543, Warsaw, Poland, Springer-Verlag (2003).

28) Nguyen, M.-H. and Vadhan, S.: Simpler Session-Key Generation from Short Random Passwords, Theory of Cryptography - TCC 2004, LNCS, Vol.2951, pp.428-445, Cambridge, MA, USA, Springer-Verlag (2004).

29) Abdalla, M., Fouque, P. and Pointcheval, D.: Password-Based Authenticated Key Exchange in the Three-Party Setting, Public Key Cryptography - PKC 2005, LNCS, Vol.3386, pp.65-84, Les Diablerets, Switzerland, Springer-Verlag (2005).

30) Abdalla, M. and Pointcheval, D.: Interactive Diffie-Hellman Assumptions with Applications to Password-Based Authentication, $\mathrm{Fi}$ nancial Cryptography and Data Security - FC 2005, LNCS, Vol.3570, pp.341-356, Roseau, The Commonwealth of Dominica, SpringerVerlag (2005).

31) Cliff, Y., Tin, Y.-S. and Boyd, C.: Password Based Server Aided Key Exchange, Applied Cryptography and Network Security (ACNS 2006), LNCS, Vol.3986, pp.146-161, Singapore, Springer-Verlag (2006).

(Received November 24, 2006)

(Accepted June 5, 2007)

(Released September 5, 2007)

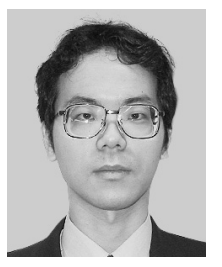

Haruki Ota received the B.E. degree, Department of Computer Science, and M.E. degree, Department of Communications and Integrated Systems, from Tokyo Institute of Technology, Japan, in 2000 and 2002 respectively. He joined KDDI and has been engaged in research on cryptographic protocol, biometrics, and information security. He is currently a research engineer of the Information Security Lab. in KDDI R\&D Laboratories Inc. He is a member of IEICE.

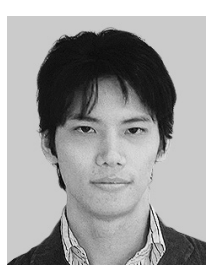

Kazuki Yoneyama received the B.E. and M.E. degrees from the University of ElectroCommunications, Tokyo, Japan, in 2004 and 2006, respectively. He is currently a doctor course student at the Graduate School of Electro-Communications since 2006. He is presently engaged in research on cryptography. $\mathrm{He}$ is a member of IEICE.

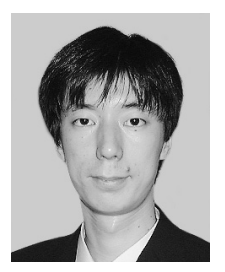

Shinsaku Kiyomoto received his B.E. degree in Engineering Sciences and his M.E. degree in Materials Science from Tsukuba University, Japan, in 1998 and 2000, respectively. He joined KDD (now KDDI), and has been engaged in research on stream ciphers, cryptographic protocols, and mobile security. He is currently a researcher of the Information Security Lab. in KDDI R\&D Laboratories Inc. He received his doctorate of engineering from Kyushu University in 2006. He received the Young Engineer Award from IEICE in 2004. He is a member of JPS and IEICE. 


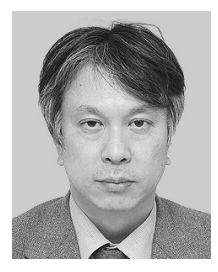

Toshiaki Tanaka received the B.E. and M.E. degrees of Communication Engineering from Osaka University, Japan, in 1984 and 1986 respectively. He joined KDD (now KDDI), and has been engaged in research on network security, cryptographic protocol, mobile security, digital rights management, and intrusion detection techniques. He is currently the leader of the Information Security Lab. in KDDI R\&D Laboratories Inc. He received his doctorate of engineering from Kyushu University in 2007. He is a member of IEICE.

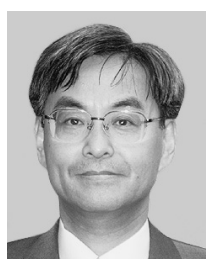

Kazuo Ohta received his B.S., M.S., and Dr.S. degrees from Waseda University, Tokyo, Japan, in 1977, 1979, and 1990, respectively. He has been a professor at the University of Electro-Communications since 2001. He was a researcher at NTT Laboratories between 1979 and 2001. He is presently engaged in research on information security. Dr. Ohta is a member of the International Association for Cryptologic Research, IEICE and IEEE. 